\title{
Diabetes mellitus im Kindes- und Jugendalter (Update 2019)
}

\author{
Birgit Rami-Merhar · Elke Fröhlich-Reiterer · Sabine E. Hofer
}

Online publiziert: 12. April 2019

(c) Der/die Autor(en) 2018

\begin{abstract}
Zusammenfassung Im Kindes- und Jugendalter ist im Gegensatz zum Erwachsenenalter der Diabetes mellitus Typ 1 (T1D) die am häufigsten auftretende Form des Diabetes mellitus ( $>95 \%)$. Nach der Diagnosestellung sollte die Betreuung der Kinder und Jugendlichen in einer Kinderabteilung mit Erfahrung in pädiatrischer Diabetologie erfolgen und nicht im niedergelassenen Bereich. Eine lebenslange Insulintherapie ist notwendig, wobei diese individuell an das Alter und den Alltag der Familie angepasst werden soll. Ein wesentlicher Teil in der Betreuung ist die Schulung von Patienten und Eltern von einem entsprechend ausgebildeten multidisziplinären Team. Die APEDÖ (Arbeitsgruppe für pädiatrische Endokrinologie und Diabetologie Österreich) hat als metabolisches Ziel für alle pädiatrischen Altersgruppen einen $\mathrm{HbA}_{1 \mathrm{c}}$-Wert $\leq 7,0 \%$ (IFCC $\leq 53 \mathrm{mmol} / \mathrm{mol}$ ) ohne schwere Hypoglykämien empfohlen. Eine altersentsprechend normale körperliche, kognitive und psychosoziale Entwicklung sowie die Vermeidung von Akutkomplikationen (schwere Hypoglykämien, diabetische Ketoazidose) und die Prävention von diabetesbedingten Spätkomplikationen zum Erhalt einer hohen Lebensqualität sind die Ziele der pädiatrischen Diabetestherapie.
\end{abstract}

\section{B. Rami-Merhar}

Universitätsklinik für Kinder- und Jugendheilkunde, Medizinische Universität Wien, Wien, Österreich

\section{E. Fröhlich-Reiterer}

Universitätsklinik für Kinder- und Jugendheilkunde,

Medizinische Universität Graz, Graz, Österreich

\section{S. E. Hofer $(\bowtie)$}

Department für Pädiatrie 1, Medizinische Universität Innsbruck, Anichstraße 35, 6020 Innsbruck, Österreich Sabine.e.Hofer@i-med.ac.at
Schlüsselwörter Typ 1 Diabetes · Metabolische Kontrolle · Schwere Hypoglykämien · Insulinpumpentherapie

\section{Diabetes mellitus in childhood and adolescence (Update 2019)}

Summary In contrast to adults, type 1 diabetes mellitus (T1D) is the most frequent form of diabetes in childhood and adolescence $(>95 \%)$. After diagnosis the management of children and adolescents with T1D should take place in highly specialized pediatric units experienced in pediatric diabetology and not in private practices. The lifelong substitution of insulin is the cornerstone of treatment whereby modalities need to be individually adapted for patient age and the family routine. Diabetes education is essential in the management of patients with diabetes and their families and needs to be performed by a multidisciplinary team consisting of a pediatric endocrinologist, diabetes educator, dietitian, psychologist and social worker. The Austrian working group for pediatric endocrinology and diabetes (APEDÖ) recommends a metabolic goal of HbAlc $\leq 7.0 \%$, International Federation for Clinical Chemistry (IFCC) $<53 \mathrm{mmol} / \mathrm{mol}$, for all pediatric age groups without the presence of severe hypoglycemia. Age-related physical, cognitive and psychosocial development, avoidance of acute diabetes-related complications (severe hypoglycemia, diabetic ketoacidosis) and prevention of diabetesrelated late complications to ensure high quality of life are the main goals of diabetes treatment in all pediatric age groups.

Keywords Type 1 diabetes · Metabolic control · Severe hypoglycemic events $\cdot$ Insulin pump treatment 


\section{Definition}

Diabetes mellitus ist eine Stoffwechselerkrankung mit unterschiedlicher Ätiologie, welche charakterisiert ist durch eine persistierende Hyperglykämie, bedingt durch eine Störung der Insulinsekretion und/oder Insulinwirkung.

\section{Klassifikation}

Die derzeit gültige Klassifikation der American Diabetes Association (ADA 2018) teilt die verschiedenen Diabetesformen in Typ I-IV ein [1]. Im Kindes- und Jugendalter tritt zu $>95 \%$ ein Diabetes mellitus Typ 1 (T1D) auf, der aufgrund des Insulinmangels rasch zu einer diabetischen Ketoazidose führen kann. Die Erstmanifestation eines T1D kann in jedem Kindes- und Jugendalter auftreten, auch im Säuglingsalter, der Erkrankungsgipfel liegt im Volkschulalter, es sind aber zunehmend immer jüngere Kinder betroffen.

Weitere im Kindes- und Jugendalter vorkommende Diabetesformen sind Diabetes mellitus Typ 2 (T2D), spezifische Diabetes-mellitus-Typen (z. B. MODY, CFrelated DM, nach Transplantation, bei Kortisontherapie) sowie assoziiert bei verschiedenen Syndromen (z.B. Trisomie 21, Prader-Willi-Syndrom u. a.).

\section{Epidemiologie}

Der T1D ist die häufigste Stoffwechselerkrankung im Kindes- und Jugendalter. Die Inzidenz der Erkrankung $<15$ Jahren nimmt auch in Österreich kontinuierlich $\mathrm{zu}[2]$.

Die aktuellsten Daten des österreichischen Diabetes-Inzidenz-Registers zur Altersgruppe 0 bis 14 Jahre stammen aus dem Jahr 2015 [3]. Im Jahr 2015 wurden in der Altersgruppe der 0- bis 14-jährigen 237 neu diagnostizierte Typ-1- sowie vier Typ-2-Diabetes-Fälle dokumentiert. Dies entspricht einer Inzidenzrate von 19,2 bzw. 0,3 Kindern pro 100.000 pro Jahr in dieser Altersgruppe. Im Beobachtungszeitraum 1999 bis 2015 gab es Schwankungen der Typ-1-Diabetes-Inzidenzrate bei Kindern mit einer jährlichen Zuwachsrate von 3,6\%. Die Inzidenzrate des Typ-2-Diabetes ist weiterhin konstant niedrig und zeigt keinen Anstieg in dieser Altersgruppe.

\section{Klinische Symptome}

Beim T1D im Kindes- und Jugendalter treten meist klassische Symptome auf wie Polyurie, Enuresis, Polydipsie, Gewichtsverlust, Müdigkeit, Konzentrationsstörungen, Sehstörungen, Verhaltensauffälligkeiten oder Soorinfektionen, wobei die Dauer dieser Symptome meist kurz (Tage bis Wochen) ist. Je jünger das Kind ist, desto schwieriger kann es sein, die Symptome einer Diabetesmanifestation zuzuordnen.
Tab. 1 Diagnosekriterien [1, 4]

$\mathrm{HbA}_{1 \mathrm{c}}>6,5 \%$ (IFCC $>48 \mathrm{mmol} / \mathrm{mol}$ ) (DCCT-standardisiertes Labor)
Oder
$\begin{aligned} & \text { Nüchtern-Plasma-Glukose } \geq 126 \mathrm{mg} / \mathrm{dl} \text { (mindestens } 8 \mathrm{~h} \text { keine Kalorienauf- } \\ & \text { nahme) } \\ & \text { Oder } \\ & \text { 2-h-Plasma-Glukose beim oGTT } \geq 200 \mathrm{mg} / \mathrm{dl} \text { (Der oGTT soll mit einer Gluko- } \\ & \text { sebelastung von } 1,75 \mathrm{~g} / \mathrm{kgKG} \text {, maximal } 75 \mathrm{~g} \text { durchgeführt werden) } \\ & \text { Oder } \\ & \text { Klassische Diabetessymptome oder hyperglykämische Krise mit einer } \\ & \text { Plasmaglukose } \geq 200 \mathrm{mg} / \mathrm{dl}\end{aligned}$

\section{Diagnosekriterien für einen Diabetes mellitus}

Die Diagnose eines T1D im Kindesalter wird meist anhand der typischen Symptome, einer Harnuntersuchung, der Blutzucker- und $\mathrm{HbA}_{\mathrm{lc}}$-Bestimmung gestellt. Es gelten in der Pädiatrie die gleichen Diagnosekriterien wie bei Erwachsenen, lediglich die Glukosebelastung beim oralen Glukosetoleranztest (oGTT) ist gewichtsbedingt unterschiedlich. Ein oGTT ist bei Kindern und Jugendlichen mit T1D nur selten notwendig, spielt aber bei der Diagnose seltenerer Diabetesformen (z.B. T2D, MODY, CF-related DM) eine wichtige Rolle (Tab. 1).

Bei klassischen Symptomen und Hyperglykämie und/oder Glukosurie/Ketonurie sollten die Kinder/ Jugendlichen umgehend an eine Kinderabteilung mit ausreichend Erfahrung in der Behandlung von Kindern mit Diabetes und Expertise in pädiatrischer Diabetestherapie überwiesen werden.

Bei Patienten mit fehlender klinischer Symptomatik, aber nachgewiesener Hyperglykämie und/oder Glukosurie (dies kann z. B. transient im Rahmen eines Infektes auftreten) sollten eine Kontaktaufnahme sowie eine weitere Abklärung in einem Zentrum für pädiatrische Diabetologie erfolgen.

\section{Therapie}

Die Betreuung und Behandlung von Kindern und Jugendlichen mit Diabetes mellitus sollte grundsätzlich in einem Zentrum für pädiatrische Diabetologie bzw. einer Kinderabteilung mit ausreichender Erfahrung in pädiatrischer Diabetologie erfolgen.

\section{Ziele}

Eine altersentsprechend normale körperliche, kognitive und psychosoziale Entwicklung sowie die Vermeidung von Akutkomplikationen (schwere Hypoglykämien, diabetische Ketoazidose) und die Prävention von diabetesbedingten Spätkomplikationen (diabetische Retinopathie, diabetische Nephropathie u.a.) zum Erhalt einer hohen Lebensqualität sind die Ziele der pädiatrischen Diabetestherapie.

Dies beinhaltet eine regelmäßige Kontrolle des Längen- und Gewichtsverlaufs, des Pubertätsstatus 
sowie die Durchführung regelmäßiger Screeninguntersuchungen auf chronische Folgeerkrankungen im Frühstadium und diabetesassoziierte Erkrankungen (Zöliakie, Schilddrüsenerkrankungen u.a.).

Die österreichische Arbeitsgruppe für pädiatrische Endokrinologie und Diabetologie (APEDÖ) fordert als Ziel für die metabolische Einstellung $\mathrm{HbA}_{1 \mathrm{c}}$-Werte $\leq 7,0 \%$ (IFCC $\leq 53 \mathrm{mmol} / \mathrm{mol}$ ) [6]. Die Empfehlungen internationaler Diabetesgesellschaften reichen von $\mathrm{HbA}_{\mathrm{lc}} \leq 6,5 \%$ (IFCC $\leq 48 \mathrm{mmol} / \mathrm{mol}$, NICE - [5]), $\leq 7,0 \%$ (IFCC $\leq 53 \mathrm{mmol} / \mathrm{mol}$, ISPAD - [6]) bis $\leq 7,5 \%$ (IFCC $\leq 58 \mathrm{mmol} / \mathrm{mol}$ ) DDG - [7] und ADA - [8]), wobei in allen genannten Gesellschaften in den letzten Jahren eine Reduktion des $\mathrm{HbA}_{\mathrm{lc}}$-Zieles vorgenommen wurde. Es gilt, den niedrigsten $\mathrm{HbA}_{\mathrm{lc}}$-Wert anzustreben, welcher ohne schwere Hypoglykämien zu erreichen ist. Höhere $\mathrm{HbA}_{\mathrm{lc}}$-Ziele können individuell bei Vorliegen einer Hypoglykämiewahrnehmungsstörung und rezidivierenden Hypoglykämien notwendig sein. Die $\mathrm{HbA}_{1 \mathrm{c}}$-Messungen sollten regelmäßig mindestens 4-mal jährlich erfolgen.

\section{Kontinuierliche Behandlung bei Diabetes mellitus Typ 1}

\section{Insulintherapie}

Eine Insulintherapie muss ab dem Zeitpunkt der Diagnosestellung eingeleitet und lebenslang fortgesetzt werden, wobei der Insulinbedarf in unterschiedlichen Phasen der Erkrankung variieren kann. Es wird empfohlen bei Kindern und Jugendlichen eine individualisierte intensivierte Insulintherapie mittels Insulinpen oder Insulinpumpe durchzuführen. Die konventionelle Therapie wird in der Pädiatrie nur noch in Einzelfällen/Sondersituationen eingesetzt.

Zur prandialen Insulinsubstitution sollten kurz wirksame Humaninsuline oder schnell wirksame Insulinanaloga, welche sich hinsichtlich ihres Wirkbeginns und der Wirkdauer unterscheiden, verwendet werden. In Insulinpumpen sollten schnell wirksame Insulinanaloga Verwendung finden [7]. Bei Kleinkindern ist gelegentlich aufgrund des geringen Insulinbedarfs die Verwendung von verdünntem Insulin (U10/U20/U50) notwendig. Die Verdünnung kann mittels physiologischer Kochsalzlösung oder mit von den Firmen zur Verfügung gestelltem Verdünnungsmedium hergestellt werden. Die Haltbarkeit der verdünnten Insuline ist begrenzt.

Als Basalinsuline werden überwiegend lang wirksame Insulinanaloga (seltener NPH-Insuline) zur Substitution des basalen Insulinbedarfs verwendet. Lang wirksame Insuline finden bei der Pumpentherapie keine Verwendung, da der Basalbedarf durch die kontinuierliche Abgabe von schnell wirksamen Insulinanaloga abgedeckt wird.

Die Insulinpumpentherapie sollte bei folgenden Indikationen eingesetzt werden [9]:
- Kleinkinder (auch Säuglinge),

- Kinder und Jugendliche mit ausgeprägtem DawnPhänomen,

- Risiko für Hypoglykämien, rezidivierende Hypoglykämien, fehlende Hypoglykämiewahrnehmung, nächtlichen Hypoglykämien,

- Nadelphobie,

- starke Blutzuckerfluktuation unabhängig vom $\mathrm{HbA}_{\mathrm{lc}}$,

- bei Vorliegen von diabetischen Spätschäden (Retinopathie, Nephropathie),

- schwangere Jugendliche.

Eine individualisierte, altersadäquate Therapie ist notwendig, um eine hohe Therapiezufriedenheit und Compliance $\mathrm{zu}$ erreichen.

\section{Glukosemessung}

Eine regelmäßige Blutzuckerselbstmessung entweder durch Blutzuckerbestimmung in kapillärem Blut aus der Fingerbeere, mittels Flash-Glukosemessung oder mittels kontinuierlicher subkutaner Glukosemessung (Real-time-CGM) sollte bei allen Kindern durchgeführt werden.

Bei der Blutzuckermessung mittels Kapillarblut ist eine Häufigkeit von 6 bis 10 Messungen täglich empfohlen. Die Messung sollte jeweils VOR den Mahlzeiten stattfinden.

Bei Verwendung der Flash-Glukosemessung sollte analog zur blutigen Messung ein Ablesen der Glukosewerte mittels Scangerät mindestens 6- bis 10-mal täglich erfolgen. Bei hohen Werten oder sehr tiefen Werten oder nicht plausiblen Werten wird die Durchführung einer blutigen Kontrollmessung empfohlen. Insbesondere wenn eine therapeutische Konsequenz abgeleitet wird, sollten blutige Kontrollmessungen angestellt werden.

Bei Verwendung von Real-time-CGM-Methoden in Zusammenhang mit Insulinpumpentherapie oder als Stand-alone-Geräte ist ein regelmäßiges Kalibrieren empfohlen. Der Zusammenschluss von Insulinpumpe mit Glukosesensoren im Sinne einer sensorunterstützten Pumpentherapie hat in den letzten Jahren in der pädiatrischen Altersgruppe stetig zugenommen. Die Möglichkeiten der Hypoglykämieabschaltung sowie der prädiktiven Hypoglykämieabschaltung sind bei Kleinkindern, Kindern mit schlechter Hypoglykämiewahrnehmung sowie bei Kindern und Jugendlichen mit schweren und/oder rekurrierenden Hypoglykämien sinnvoll (s. auch ÖDG Leitlinie Kontinuierliche Glukosemessung bei Diabetes mellitus).

Eine regelmäßige Dokumentation der gemessenen Zuckerwerte, Insulindosen und Broteinheiten erscheint im Alltag hilfreich. Alle diabetesrelevanten Geräte (Pumpe, Blutzuckermessgeräte, Sensoren) sollten im Rahmen der ärztlichen Kontrolle ausgelesen und standardisiert ausgewertet und analysiert 
werden, um therapeutische Maßnahmen setzen $\mathrm{zu}$ können.

\section{Therapie bei Diabetes mellitus Typ 2 (T2D)}

Zur Therapie des T2D wird primär eine Lebensstilmodifikation angestrebt, an Medikamenten sind derzeit nur Metformin (ab dem 10. Lebensjahr) und Insulin zugelassen, es gibt zurzeit noch keine einheitlichen pädiatrischen Empfehlungen. Zahlreiche neuere Medikamente, welche bei Erwachsenen mit T2D eingesetzt werden, sind für die pädiatrische Altersgruppe noch nicht erprobt/zugelassen.

\section{Ernährung}

Die Ernährungsschulung und das Einhalten einer kohlenhydratberechnenden Kost sind eine wichtige Grundvoraussetzung für eine gute metabolische Einstellung. Die Schulung über die Berechnung der Nahrung (insbesondere der Kohlenhydrate) und deren Wirkung auf den Blutzucker sollte von DiätologInnen durchgeführt werden. Im Rahmen der Schulung sollte auf kulturelle Ernährungsgewohnheiten Rücksicht genommen werden.

Neben einer altersgerechten Kalorien- und Energiezufuhr soll ein ausreichender Anteil an Kohlenhydraten (50-55\%) zur Sicherstellung eines regulären altersentsprechenden Wachstums zugeführt werden [10].

\section{Schulung}

Eine altersangepasste, strukturierte Diabetesschulung ist integrativer Bestandteil der therapeutischen Bemühungen und Voraussetzung für ein funktionierendes Diabetesmanagement zu Hause. Die Diabetesschulung umfasst alle in dieser Behandlungsempfehlung aufgelisteten Aspekte der pädiatrischen Diabetestherapie.

\section{Psychologische Interventionen}

Das multidisziplinäre Behandlungsteam sollte durch eine/n Psychologen/in unterstützt werden, welche/r bei der Erfassung der psychosozialen Situation der Patienten/Familien im Rahmen dieser chronischen Erkrankung eine wichtige Rolle einnimmt und ggf. auch spezifische Interventionen durchführen kann. Der Erstkontakt mit Kind und Familie erfolgt idealerweise während der Manifestation.

Zahlreiche psychiatrische Komorbiditäten sind bei T1D bekannt (Essstörungen, Insulinmanipulation, Depression, Angststörung, ADHS u.a.).

\section{Akute Komplikationen}

Zu den Akutkomplikationen des T1D zählen zum einen die schwere Hypoglykämie und zum anderen die diabetische Ketoazidose (DKA).
Die schwere Hypoglykämie ist definiert als ein Ereignis mit Bewusstseinsbeeinträchtigung (Koma oder Krampfanfall), das einer Fremdhilfe bedarf.

Die Behandlung erfolgt im Homesetting mit Glucagon (0,5-1 mg) i.m. oder s.c. bzw. im Kliniksetting mit Glucose i.v. (2-3 ml/kgKG 10\% Glucose). Die Glucagon-Fertigspritze sollte in jedem Haushalt mit einem Kind mit T1D vorhanden sein. Der Einsatz der Glucagon-Notfallspritze setzt eine entsprechende Schulung voraus [11]. Andere Applikationsarten (nasales Glucagon) sind derzeit in Österreich noch nicht erhältlich.

Kleinkinder sind aufgrund der mangelnden Fähigkeit, Hypoglykämiesymptome zu äußern, und durch eine verminderte Hypoglykämiewahrnehmung einem höheren Risiko für das Auftreten einer schweren Hypoglykämie ausgesetzt.

Internationale Registerstudien konnten zeigen, dass die Inzidenz der schweren Hypoglykämien in den letzten 2 Dekaden abgenommen hat. Eine Korrelation von niedrigen $\mathrm{HbA}_{1 \mathrm{c}}$-Werten und damit verbunden höherer Hypoglykämierate konnte nicht bestätigt werden [12].

Eine weitere akute Komplikation ist die diabetische Ketoazidose (DKA). In Österreich wurde im Zeitraum 1989 bis 2008 bei mehr als einem Drittel der Patienten im Rahmen der Diabetesmanifestation eine Ketoazidose festgestellt $[13,14]$.

Die klinischen Zeichen einer DKA sind: Dehydratation, Tachykardie, Tachypnoe, Kußmaul-Atmung, Azetongeruch, Übelkeit und Erbrechen bis hin zum Koma.

Risikofaktoren für eine DKA bei Erstmanifestation sind: jüngeres Alter, verzögerte Diagnosestellung, niedriger sozioökonomischer Status und Länder mit niedriger Diabetesinzidenz. Risikofaktoren für eine DKA bei bekanntem T1D sind v.a. absichtliches Weglassen der Insulininjektionen, Fehlverhalten bei Krankheit und Fehlverhalten bei Insulinpumpentherapie.

Die Therapie und Überwachung von Kindern mit DKA sollte grundsätzlich von Ärzten durchgeführt werden, die über Erfahrung in diesem Gebiet verfügen, und es muss die Möglichkeit $\mathrm{zu}$ einer intensivmedizinischen ärztlichen, pflegerischen und biochemischen Überwachung gesichert sein.

Die Therapieziele der DKA sind: Ausgleich der Dehydratation, Ausgleich der Azidose, Blutzuckerstabilisierung und -normalisierung und Vermeidung von Komplikationen (Hirnödem und Hypokaliämie).

Die Vermeidung von akuten Komplikationen zählt zu den vorrangigen Zielen in der Betreuung von Kindern und Jugendlichen mit T1D.

\section{Langzeitkomplikationen und Screening- untersuchungen}

Bei den Follow-up-Untersuchungen sollen routinemäßig die Körperlänge, das Gewicht, der Blutdruck, das Pubertätsstadium sowie die Injektionsstellen bzw. 
Katheterinjektionsstellen (cave Lipohypertrophien/ Lipoatrophien und Hautirritationen) und CGM-Stellen (Ekzeme/Hautirritationen/Abszesse) kontrolliert werden.

Einmal im Jahr ist auch die Kontrolle der Nierenund Leberfunktionsparameter, des Blutbildes sowie des Lipidstatus indiziert. Der $\mathrm{HbA}_{\mathrm{lc}}$ sollte alle 3 Monate bestimmt werden.

Um das Risiko für mikrovaskuläre und makrovaskuläre Komplikationen zu senken, sollte eine möglichst normoglykäme Stoffwechseleinstellung angestrebt werden, um das Auftreten von diabetischen Komplikationen wie eine diabetische Retinopathie [14, 15], eine Mikroalbuminurie [16, 17] oder eine Hypertonie [18] bei Jugendlichen zu verhindern.

Ein jährliches Screening auf mikrovaskuläre Komplikationen wie Nephropathie (Morgenharn: Albumin/Kreatinin-Ratio), Retinopathie (Fundoskopie) und Neuropathie (klinische Untersuchung auf Sensibilität, Vibrationsempfinden und Reflexe) wird jährlich ab dem 11. Lebensjahr und 2 bis 5 Jahren Diabetesdauer empfohlen. Bei einer Diabetesdauer $<10$ Jahren und guter Stoffwechselkontrolle scheint ein 2-jähriges Retinopathiescreening ausreichend zu sein.

Ein Screening auf makrovaskuläre Komplikationen soll nach dem 11. Lebensjahr begonnen werden (jährlich RR, alle 5 Jahre Lipidstatus) [19].

Bei Verdacht auf eine Hypertonie sollte eine 24-hBlutdruckmessung durchgeführt werden (unter Verwendung alters- und geschlechtsspezifischer Normwerte) $[20,21]$. ACE-Hemmer werden zur Senkung eines erhöhten Blutdrucks im Kindes- und Jugendalter empfohlen und haben sich als sichere und effektive Therapie erwiesen. Der klinische Benefit von Angiotensin-II-Rezeptorantagonisten ist ähnlich wie bei ACE-Hemmern, wobei ihre Verwendung in der Kinder- und Jugendheilkunde nicht so weit verbreitet ist. Des Weiteren vermindern ACE-Hemmer die Progression von einer Mikroalbuminurie zur Makroalbuminurie und erhöhen die Regressionsrate zur Normoalbuminurie [21].

\section{Assoziierte Erkrankungen}

Kinder und Jugendliche mit T1D haben ein höheres Risiko, weitere Autoimmunerkrankungen zu entwickeln.

Bis zu $29 \%$ der Patienten mit T1D entwickeln positive Schilddrüsenantikörper (Autoimmunthyreoiditis - AIT); 3-8\% der Jugendlichen haben eine Hypothyreose. Die AIT ist die häufigste assoziierte Autoimmunerkrankung und kommt häufiger bei Mädchen vor, oft manifestiert sie sich in der Pubertät, und sie ist mit einer längeren Diabetesdauer und dem Alter assoziiert [22].

Ein Screening auf assoziierte AIT (mittels TSH, fT4, fT3 und TPO $^{-}$und Tg AK) wird bei Erstmanifestation und danach alle 2 Jahre bei asymptomatischen Patienten empfohlen.
Die Zöliakie (CD) wird bei 1-10\% der Kinder mit T1D diagnostiziert, wobei Kinder mit sehr jungem Diabetesmanifestationsalter ein höheres Risiko für die Entwicklung einer Zöliakie haben. Die klassischen Symptome der Zöliakie, wie z. B. Gedeihstörung und aufgetriebenes Abdomen, sind eher selten, meist sind die Patienten asymptomatisch. Ein Screening auf Zöliakie (mittels EMA, tTG und zusätzlicher Ausschluss eines IgA-Mangels) wird bei Erstmanifestation und danach nach 2 Jahren und 5 Jahren empfohlen. Bei klinischen Symptomen oder erstgradigen Verwandten mit CD sollte öfter gescreent werden. HLA-DQ2 und HLA-DQ8 sind bei Patienten mit T1D häufig positiv und werden deshalb nicht als Screeninguntersuchung empfohlen.

Kinder mit positiven CD-Antikörpern sollen gastroenterologisch untersucht und das Vorliegen einer Zöliakie soll bioptisch gesichert werden.

Weitere Autoimmunerkrankungen wie Hyperthyreose, Nebenniereninsuffizienz, Vitiligo oder Polyendokrinopathien und Autoimmungastritis sind seltener. Bei Symptomen sollten Diabetologen auch an die Möglichkeit dieser selteneren Autoimmunerkrankungen denken.

\section{Transition}

Im Alter von 18 bis 19 Jahren bzw. mit Abschluss der Schulausbildung/Lehre sollten die Patienten an die Erwachsenenmediziner übergeben werden. Diese Transition soll flexibel, je nach „Reife“ des Jugendlichen, geordnet und im Idealfall im Rahmen einer Transitionsklinik erfolgen. Die Transition sollte möglichst frühzeitig besprochen und individuell geplant werden, und es sollte keine „Lücke“ in der Betreuung entstehen. Spezielle Transitionskliniken und internistische Kliniken, die auf die Bedürfnisse der Jugendlichen eingehen und bei denen eine Verbindung zwischen pädiatrischem und internistischem Zentrum besteht, haben sich sehr bewährt.

Rückmeldungen nach erfolgreicher Transition an den Pädiater sind wünschenswert.

Funding Open access funding provided by University of Innsbruck and Medical University of Innsbruck.

Interessenkonflikt B. Rami-Merhar hat in den letzten 36 Monaten von folgenden Firmen Honorare/Forschungsgelder erhalten: Eli Lilly, Medtronic, Novo Nordisk, Menarini, Roche. E. Fröhlich-Reiterer hat in den letzten 36 Monaten von folgenden Firmen Honorare/Forschungsgelder erhalten: Medtronic, Novo Nordisk, Eli Lilly, Roche. S.E. Hofer hat in den letzten 36 Monaten von folgenden Firmen Honorare/ Forschungsgelder erhalten: Eli Lilly, Sanofi.

Open Access Dieser Artikel wird unter der Creative Commons Namensnennung 4.0 International Lizenz (http:// creativecommons.org/licenses/by/4.0/deed.de) veröffentlicht, welche die Nutzung, Vervielfältigung, Bearbeitung, Verbreitung und Wiedergabe in jeglichem Medium und Format erlaubt, sofern Sie den/die ursprünglichen Autor(en) und die Quelle ordnungsgemäß nennen, einen Link zur Creative 
Commons Lizenz beifügen und angeben, ob Änderungen vorgenommen wurden.

\section{Literatur}

1. American Diabetes Association. Classification and diagnosis of diabetes. Standards of medical care in diabetes-2018. Diabetes Care. 2018;41(Suppl.1):S13-S27.

2. Schober E, Waldhoer T, Rami B, Hofer S. Incidence and time trend of type 1 and type 2 diabetes in Austrian children 1999-2007.JPediatr. 2009;155:190.https://doi.org/10. 1016/j.jpeds.2009.03.010.

3. Rami-Merhar B, Hofer S, Fröhlich-Reiterer E, Fritsch M, Waldhör T. Incidence trends of type 1 and type 2 diabetes in Austrian children $<15$ years (1999-2015). Paediatr Diabetes. 2016;24(Suppl 17):94.

4. Mayer-Davis EJ, Kahkoska AR, Jefferies C, Dabelea D, Balde N, Gong CY, Aschner P, Craig ME. Definition, epidemiology and classification of diabetes in children and adolescents. 2018 ISPAD Clinical Practice Consensus Guidelines. Pediatr Diabetes. 2018;19:7.https://doi.org/10.1111/pedi.12773.

5. Beckles ZL, Edge JA, Mugglestone MA, Murphy MS, Wales JK, Guideline Development G. Diagnosis and management of diabetes in children and young people: summary of updated NICE guidance. BMJ. 2016;352:i139. https://doi. org/10.1111/pedi.12773.

6. DiMeglio LA, Acerini CL, Codner E, Craig ME, Hofer SE, Pillay K, Maahs D. Glycemic control targets and glucose monitoring for children and adolescents with type 1 diabetes. 2018 ISPAD Clinical Practice Consensus Guidelines. Pediatr Diabetes. 2018; https://doi.org/10.1111/ped.12737.

7. NeuA, Bürger-Büsing J, DanneT, DostA, Holder M, Holl RW, Holterhus PM, Kapellen T, Karges B, Kordonouri O, Lange K, Müller S, Raile K, Schweizer R, Sengbusch S, Stachow R, Wagner V, Wiegand S, Ziegler R. Diagnostik, Therapie und Verlaufskontrolle des Diabetes mellitusim Kindes-und Jugendalter. Diabetologie. 2017;12(Suppl2):S190-S202.

8. American Diabetes Association. Children and adolescents. Diabetes Care. 2017;40(Suppl. 1):S105-S13.

9. Phillip M, Battelino T, Rodriguez H, Danne T, Kaufman F, European Society for Paediatric Endocrinology, Lawson Wilkins Pediatric Endocrine Society, International Society for Pediatric and Adolescent Diabetes, American Diabetes Association, European Association for the Study of Diabetes. Diabetes Care. 2007;30(6):1653-62.

10. Smart CE, Annan F, Higgins LA, Jelleryd E, Lopez M, Acerini CL. Nutritional management in children and adolescents with diabetes. ISPAD Clinical Practice Consensus Guidelines 2018. Pediatr Diabetes. 2018; https://doi.org/10.1111/ pedi.12738.

11. Abraham MB, Jones TW, Naranjo D, Karges B, Oduwole A, Tauschmann M, Maahs D. Assessment and management of hypoglycemia in children and adolescents with diabetes. ISPAD Clinical Practice Consensus Guidelines 2018. Pediatr Diabetes. 2018; https://doi.org/10.1111/pedi.12698.
12. Karges B, Schwandt A, Heidtmann B, Kordonouri O, Binder E, Schierloh U, Boettcher C, Kapellen T, Rosenbauer J, Holl RW. Association of insulin pump therapy vs insulin injection therapy with severe hypoglycemia, ketoacidosis, and glycemic control among children, adolescents, and young adults with type 1 diabetes. JAMA. 2017;318(14):1358-1313.

13. Schober E, Rami B, Waldhoer T. Diabetic ketoacidosis at diagnosis in Austrian children in 1989-2008: a populationbased analysis. Diabetologia.2010;53:1057-61.

14. Fritsch M, Schober E, Rami-Merhar B, Hofer S, FröhlichReiterer E, Waldhoer T. Diabetic ketoacidosis at diagnosis in Austrian children: a population-based analysis, 1989-2011. JPediatr. 2013;163(5):1484.

15. The Diabetes Control and Complications Trial Research Group. The effect of intensive treatment of diabetes on the development and progression of long-term complications in insulin-dependent diabetes mellitus. N Engl J Med. 1993;329(14):977-86.

16. Hofer SE, Raile K, Fröhlich-Reiterer E, Kapellen T, Dost A, Rosenbauer J, et al. Tracking of metabolic control from childhood to young adulthood in type 1 diabetes. J Pediatr. 2014;165(5):956-961.e2.

17. Hofer SE, Raile K, Fröhlich-Reiterer E, Kapellen T, Dost A, Rosenbauer J, Grulich-Henn J, Austrian/German Diabetes Patienten Verlaufsdokumentation DPV Initiative, German Competence Network for Diabetes Mellitus.. Tracking of metabolic control from childhood to young adulthood in type 1 diabetes. J Pediatr. 2014;165(5):956-61.

18. Raile K, Galler A, Hofer S, Herbst A, Dunstheimer D, Busch PHR. Diabetic nephropathyin 27,805 children, adolescents, and adults with type 1 diabetes: effect of diabetes duration, A1C, hypertension, dyslipidemia, diabetes onset, and sex. Diabetes Care. 2007;30:2523-8.

19. Schultz CJ, Neil HA, Dalton RN, Konopelska Bahu T, Oxford Regional Prospective Study Group. Blood pressure does not rise before the onset of microalbuminuria in children followed from diagnosis of type 1 diabetes. Oxford Regional ProspectiveStudyGroup.DiabetesCare.2001;24(3):555-60.

20. Donaghue KC, Marcovecchio L, Wadwa RP, Chew EY, Wong T, Calliari E, Zabeen B, Salem MM, Craig ME. Microvascular and macrovascular complications in children and adolescents. ISPAD Clinical Practice Consensus Guidelines 2018. Pediatr Diabetes. 2018; https://doi.org/10.1111/pedi. 12742.

21. National High Blood Pressure Education Program Working Group on High Blood Pressure in Children and Adolescents. The fourth report on the diagnosis, evaluation, and treatment of high blood pressure in children and adolescents. Pediatrics. 2004;114(2 Suppl4th Report):555-76.

22. Mahmud FH, Elbarbary NS, Fröhlich-Reiterer E, Holl RW, KordonouriO, Knip M,Simmons K, CraigME. Other complications and diabetes-associated conditions in children and adolescents. ISPAD Clinical Practice Consensus Guidelines 2018. Pediatr Diabetes. 2018;31 https://doi.org/10.1111/ pedi. 12740 . 